\title{
Stalk versus base invasion in pT1 papillary cancers of the bladder: improved substaging system predicting risk of progression
}

\author{
Margaret Lawless, MD $^{1}$, Roman Gulati ${ }^{2}$, and Maria Tretiakova, MD, PhD ${ }^{1}$ \\ ${ }^{1}$ Department of Pathology, University of Washington School of Medicine, Seattle, WA \\ ${ }^{2}$ Fred Hutchison Cancer Research Center, Seattle, WA
}

\begin{abstract}
Aims-Pathologic stage T1 (pT1) bladder cancers are a clinically heterogeneous group. However, current staging guidelines for superficially invasive cancers do not acknowledge the variability in type and extent of lamina propria invasion in papillary urothelial carcinomas (PUC), and historically proposed substaging systems showed either high inter-observer variation or limited value in predicting patient outcomes. Herein we reappraise pT1 PUC substaging, aiming to identify a novel scheme which is reproducible and prognostically meaningful.
\end{abstract}

Methods and results-Stage pT1 PUC diagnosed during years 1999-2015 were retrospectively reviewed and characterized as focal invasion confined to papillary stalk, focal invasion of tumor base, or extensive invasion of tumor base. Cases with concurrent flat carcinoma in-situ, angiolymphatic invasion, absent muscularis propria, or clinically advanced disease were excluded. We calculated cumulative incidence of recurrence, progression, and death by tumor subtype and evaluated differential risks using log-rank tests and Kaplan-Meier curves stratified by type and extent of invasion. Among 62 patients satisfying inclusion criteria, 22 of 29 patients with base extensive invasion progressed while 4 of 13 with base focal and 0 of 20 with stalk only invasion progressed. There was strong evidence that base extensive patients had higher risk of progression and death due to bladder cancer than base focal or stalk only counterparts $(\mathrm{P}<0.0001)$. However, tumor subtype was not significantly associated with risk of recurrence $(\mathrm{P}=0.21)$.

Conclusions-We propose an innovative substaging approach for reporting site and extent of lamina propria invasion in patients with pT1 PUC allowing patient stratification for risk of progression.

Please address correspondence to: Maria Tretiakova, MD, PhD, Department of Pathology, University of Washington School of Medicine, 325 9th Ave, Seattle, WA 98104, Box 359791, Telephone: 206-897-4294, Fax: 206-744-4343, mariast@uw.edu.

Conflicts of interest

The authors declare no conflicts of interest.

Author contributions

Margaret Lawless: review of patient records, case selection and review, data analysis and interpretation, manuscript writing and revision.

Roman Gulati: statistical analysis and data interpretation, manuscript review and revision.

Maria Tretiakova: study design, case review and classification, data analysis and interpretation, manuscript writing, review, and revision. 


\section{Keywords}

Papillary urothelial carcinoma; stalk invasion; bladder

\section{INTRODUCTION}

Three-quarters of newly diagnosed cases of bladder tumors are exophytic papillary urothelial carcinomas (PUC) that are either confined to the epithelium (stage Ta) or invade only into the lamina propria (stage T1) [1]. These tumors are generally managed with transurethral resection, with or without adjuvant intravesical therapy [2]. However, approximately 50$70 \%$ of patients with such tumors have a recurrence or a new urothelial carcinoma in the bladder within 5 years [3]. Although stage T1 (pT1) bladder cancers are heterogeneous with respect to outcomes, current guidelines for superficially invasive cancers do not discriminate between different levels of lamina propria invasion $[4,5]$.

Several substaging systems were proposed but showed high inter-observer variation, lack of consensus on criteria, and inability to segregate lesions which focally and superficially invade the papillary stalk [1, 6-12]. Some studies have shown improved survival for patients with $\mathrm{T} 1$ lesions treated by immediate cystectomy [13-15], but others have not shown a benefit [16]. Thus, there remains an interest in identifying high-risk patients within the T1 group that would benefit most from cystectomy.

The majority of PUC of the bladder have a stalk composed of loose connective tissue with fine capillaries, some inflammatory cell infiltrate, and edema, which has a distinct appearance histologically and on magnetic resonance imaging [17]. The composition of tumor base differs from stalk due to overgrowth of denser fibrous tissue, presence of pulledin superficial muscular bundles, increased fibroblastic proliferation, and inflammation of adjacent thickened bladder wall [17]. We employed an innovative practical approach to papillary pT1 substaging by separating invasion of the lamina propria into the stalk from base invasion, either focal or extensive, and evaluated outcomes over moderate follow-up.

\section{MATERIAL AND METHODS}

\section{Case selection}

The pathology archives of the University of Washington Medical Center (UWMC) were searched for primary PUC diagnoses during the period 1999-2015, yielding 285 transurethral resection bladder tumors (TURBT) with either definite lamina propria invasion (pT1) or suspicion for lamina propria invasion (pTa or pT1). After database analysis and slide review by the primary pathologist (ML), cases with predominantly non-papillary configuration, concurrent flat carcinoma in-situ (CIS), angiolymphatic space invasion, micropapillary features, absent muscularis propria, prior intravesical therapy, or clinically advanced disease were excluded. Sixty-two cases satisfied selection criteria, were independently re-reviewed by a specialized genitourinary pathologist (MT), and divided into three pT1 groups: (1) invasion confined to papillary stalk ( $\mathrm{N}=20,32 \%)$, (2) focal invasion of tumor base $(\mathrm{N}=13,21 \%)$, or (3) extensive (multifocal) invasion of tumor base $(\mathrm{N}=29,47 \%)$. 
Morphologic features of stalk only invasive PUC were similar to previously described [18] with either unifocal or multifocal involvement of edematous loose connective stroma of papillary stalks free of muscle fascicles, dense collagen, vascular plexus, and cautery effect (Figure 1). Base invasion was divided into focal and extensive categories using previously proposed morphologic criteria [8] with modification tested in a larger scale recent multiinstitutional study [1]. The base focal category is defined as either a single focus of microinvasion size smaller than $1 \mathrm{~mm}$ or more than one microinvasive foci all visible within 1 high power field of view (HPF) at $20 \times$ corresponding to $1 \mathrm{~mm}$ area. The base extensive category is defined as either a single microinvasive focus larger than $1 \mathrm{~mm}$ in size or two or more foci of invasion that are more than $1 \mathrm{~mm}$ from each other or present in multiple tissue fragments.

All patients were treated and followed-up at a single academic institution (UWMC) in accordance with the American Urological Association guidelines for non-muscle invasive bladder cancer. The treatment included intravesical therapy for the majority of patients (60\%) with bacillus Calmette-Guerin (BCG) immunotherapy (70\%) or mitomycin (30\%), reTURBT (8\%), partial or total cystectomy (26\%), and active surveillance (6\%) due to other co-morbidities, including coagulopathy, prior history of tuberculosis, and frequent infections. The most common approach was patient re-assessment with urine cytology and cystoscopy every 3 months in the first 2 years after initial diagnosis followed by every 6 months for the subsequent 2 years, and then annually thereafter (https://www.auanet.org/ education/guidelines/bladder-cancer.cfm).

\section{Statistical analysis}

Differences in patient and tumor characteristics across tumor subtypes were evaluated using the Kruskal-Wallis test for continuous characteristics or Fisher's exact test for categorical characteristics. Recurrence was defined as the reappearance of a histologically confirmed non-muscle invasive papillary urothelial carcinoma (including pTa or pT1). Recurrence-free survival (RFS) was defined as time from primary treatment to first documented evidence of any non-muscle invasive papillary cancer with censoring at the first progression event, othercause death, or last follow-up. Progression was defined as development of muscle invasive (pT2) or higher stage carcinoma, distant metastasis, or multifocal refractory to treatment pT1 disease with CIS. Progression-free survival (PFS) was defined as time from primary treatment to first document progression event with censoring at other-cause death or last follow-up. Event-free survival (EFS) was defined as time from primary treatment to the earlier of first documented progression event or first documented recurrence event with censoring at other-cause death or last follow-up. Cause-specific survival (CSS) was defined as time from primary treatment to death due to bladder cancer with censoring at other-cause death or last follow-up. Overall survival (OS) was defined as time from primary treatment to death from any cause with censoring at last follow-up. Survival endpoints were evaluated across tumor subtypes, between patients with age at diagnosis above and below the median age, and between patients with tumor size above and below the median tumor size, and differential risks were evaluated using Kaplan-Meier estimation and log-rank tests. A subset analysis repeated these estimations and tests excluding low-grade cases. Univariate Cox proportional hazards models were fitted to evaluate associations between tumor subtypes 
and the survival endpoints. Cumulative incidence of follow-up procedures for progression, types of progression, types of recurrence, and causes of death were calculated across tumor subtypes using the R package cmprsk (Gray, B. cmprsk: Subdistribution analysis of competing risks: $\mathrm{R}$ package version 2.2-7; http://cran.r-project.org/package=cmprsk). Bonferroni adjustment was made for tests involving these 3 endpoints; consequently, only pvalues $<0.05 / 3=0.017$ were considered statistically significant.

\section{Ethics}

All research was performed with the approval of the Fred Hutchinson Cancer Research Center and UWMC Institutional Review Board (IRB\#52389, 08/03/2016). This retrospective study utilizing existing human specimens, data and clinical information did not require contact of participants thus was approved with waiver of consent as per guidelines of UWMC Human Subjects Division.

\section{RESULTS}

Sixty-two patients with primary pT1 PUC met all selection criteria and included 48 (77\%) male and 14 (23\%) female patients. The median age at diagnosis was 69 (range 44-95) years with some variation across the patient groups. A total of 20 patients met criteria for stalk only, 13 for base focal, and 29 for base extensive (Table 1, Figure 1). Concomitant stalk invasion was noted in two patients with base focal invasion and two patients with base extensive invasion. On average, patients with stalk only invasion were 4 years older than patients with focal base invasion and 9 years older than patients with base extensive invasion. Median follow-up was 3.2 (range 0.1-16.7) years. The majority of patients were white ( $\mathrm{N}=56,90 \%)$, while $3(5 \%)$ were Asian/multi-ethnic, 2 (3\%) were black, and $1(2 \%)$ of unknown race. The majority of T1 PUC $(\mathrm{N}=57,92 \%)$ were high-grade tumors with the remaining 5 (8\%) cases low-grade, 4 of which showed stalk only invasion. The maximum dimension of tumor averaged 2 (range $0.3-9.0$ ) $\mathrm{cm}$. Table 1 summarizes patient and tumor characteristics for the 62 patients grouped into pT1 subtypes. There was no evidence that clinico-pathological characteristics significantly differed across tumor subtypes.

All patients were followed by urine cytology with cystoscopy ( $\mathrm{N}=59)$ and follow-up procedures including re-biopsies $(\mathrm{N}=18)$, re-TURBT $(\mathrm{N}=12)$, partial cystectomies $(\mathrm{N}=5)$, cystectomies $(\mathrm{N}=18)$, or lymph node biopsy $(\mathrm{N}=1)$. Cumulative incidence functions (CIFs) for recurrence, progression, and death with estimated values over 9 years of follow-up are reported in Table 2 and illustrated by tumor subtype in supplemental Figure 1. Across subtypes, recurrence was observed in 11 patients (CIF 26\%) and progression in 29 patients (CIF 51\%). There was strong evidence that pT1 subtype was associated with risk of progression $(\mathrm{P}<0.0001)$, but no evidence of association with risk of recurrence $(\mathrm{P}=0.21)$. More specifically, recurrence was observed in 4 (CIF 30\%) of stalk only patients, 4 (CIF $51 \%$ ) of base focal, and only 3 (CIF 10\%) of the base extensive patient cohort, which had the highest incidence of cystectomies (CIF 48\%). None of pT1 base focal recurred with a higher substage of pT1 base extensive. Across subtypes, progression events included base extensive multifocal pT1 with or without concomitant CIS ( $\mathrm{N}=8,14 \%), \mathrm{pT} 2(\mathrm{~N}=13,23 \%)$, pT3 $(\mathrm{N}=4,7 \%)$, pT4 $(\mathrm{N}=2,3 \%)$, and metastatic disease $(\mathrm{N}=2,4 \%)$. Strikingly, $86 \%$ of base 
extensive patients versus $33 \%$ of base focal patients experienced progression within 9 years of follow-up. There were no progression events in the stalk invasive group. Over 9 years of follow-up, there were no deaths due to bladder cancer among stalk only patients, 1 death (CIF 13\%) in base focal patients, and 4 deaths (CIF 16\%) among base extensive patients, showing statistically significant risk of cancer-specific mortality for the base extensive group. Furthermore, there were 3 (CIF 30\%), 4 (CIF 54\%), and 7 (CIF 41\%) deaths due to other causes, respectively.

Kaplan-Meier curves and log-rank tests provided strong evidence that progression-free survival (PFS) and event-free survival differed across subtypes (both $\mathrm{P}<0.0001$ ) (Figure 2). Median PFS in the base extensive patients was 4.1 (95\% confidence interval [CI] 3.6 to 14.3) months. Median PFS in the other 2 groups was not achieved. In contrast, there was no evidence of different recurrence-free survival across subtypes $(\mathrm{P}=0.43)$. Overall survival did not differ across subtypes $(\mathrm{P}=0.61)$. Exploratory analysis of risks of recurrence or progression by age at diagnosis above or below the median age did not show a significant difference $(\mathrm{P}=0.44$ or $\mathrm{P}=0.49$, respectively). Similarly, the size of tumor above or below the median value of $1.4 \mathrm{~cm}$ was not associated with risks of recurrence or progression $(\mathrm{P}=0.99$ or $\mathrm{P}=0.25$, respectively). In our subset analysis, excluding the low-grade cases and repeating the Kaplan-Meier estimations and CIF analyses did not materially affect our conclusions because 4 out of the 5 low-grade cases were stalk only subtype, which had few events (data not shown).

Univariate Cox model analyses showed that extent of lamina propria invasion had statistically significant impact on tumor recurrence and/or progression with hazard ratio 2.8 in base focal vs 7.3 in base extensive ( $\mathrm{P}=0.0002$, Table 3$)$.

\section{DISCUSSION}

We found that the proposed subtyping of papillary urothelial carcinomas into stalk invasive, base focal, and base extensive groups yielded a promising and practical system for stratifying patients that corresponds with the risk of progression. The greatest benefit of pT1 subtyping was its ability to stratify patients with respect to progression-free and event-free survival $(\mathrm{P}<0.0001)$. In our study, the incidence of progression and cancer-specific mortality were lowest in stalk only and highest in base extensive invasion groups. While we found no evidence that subtypes were associated with recurrence or overall survival, this is perhaps unsurprising given the older age of patients with stalk only invasion in our study, the limited number of patients, and the overall favorable survival for pT1 patients under moderate follow-up. Our findings are consistent with a recent large-scale meta-analysis and prospective studies showing significant association of pT1 substaging with progression and cancer-specific survival but not recurrence $[15,19]$.

Lamina propria (LP) invasion (pathologic stage pT1) remains an area of great interest and controversy because it adds significantly to the risks of recurrence and progression to muscle invasive disease $[2,15]$. Accumulating data suggest that substaging of pT1 disease is clinically relevant [15], but there is no agreement about the optimal approach. Several systems have been generated to subclassify the LP involvement by level, depth, and extent of 
involvement. These proposals include substaging into pT1 using muscularis mucosa (MM) and the associated large blood vessels as landmarks [7, 9, 19, 20]. However, due to high individual variation in MM and LP vascular plexus; difficulty in pathologic interpretation of small, fragmented, poorly oriented, or cauterized tissue; and no identifiable MM in a high percentage of cases, especially in PUC stroma, this approach is highly problematic $[2,7,11$, $18,21]$. Measuring depth of tumor invasion using a micrometer is another approach originally promoted by Cheng and co-authors [6]. Upon validation, these methods yielded numerous cut-off points of $0.5,1.0,1.5,3.0$, and $6.0 \mathrm{~mm}$, all claimed to be significantly associated with outcomes [2, 6, 10, 18, 22]. Other morphometric measurements, such as tumor diameter and aggregate length, were also suggested as prognostic factors with classifications of $<3,3-5$, and $>5$ or $>6 \mathrm{~mm}$ as significant prognostic variables [10]. Apparently, an objective measuring approach is also not without the significant drawbacks of variable thresholds and cumbersome morphometric assessment, and thus unlikely to gain wide acceptance.

The novelty of our approach is in separating stalk invasion from base invasion, and further case stratification by invasive tumor extent in PUC. Stalk invasion is viewed as an early stage of tumor invasion, where cells are penetrating into the morphologically distinct, loose, often edematous, connective stroma. These invasive cells and tumor nests are still confined within exophytic tumor component at a significant distance from the proper bladder wall and associated deeper vasculature, and thus less likely to spread and progress than tumors invasive into the PUC base. The significance of separating stalk invasive tumors was recognized earlier in a few studies but lacked a systematic approach and strict selection criteria [7, 23, 24]. For instance, studies by Hermann and Tommasini-Degna [7, 23] included in their cohorts cases with associated CIS, thus increasing numbers of patients but potentially affecting outcomes. The T1 tumors were substaged into T1a - invasion of the tumor stalk without involvement of LP under the stalk, stage T1b - LP invasion under the tumor stalk but superficial to MM, and stage T1c - invasion into or deeper than MM without involvement of MP [7]. The problem with this approach was lack of MM in about 50\% of their cases. Despite that, pathologic upstaging to pT2 disease did not occur in cases with stalk only invasion compared to cases with "under the stalk invasion" [7]. For base invasion, we modified the previously tested $\mathrm{T} 1 \mathrm{~m} / \mathrm{e}$ system of separating focal microinvasive (m) tumors from extensive (e) categories using rather simple morphologic criteria of $20 \times \mathrm{HPF}$ equivalent to $1 \mathrm{~mm}$ invasion [1, 2, 8, 25]. This system is very practical, could be applied to virtually every case, and proved reproducible in $81-100 \%$ of the tumors $[1,8,22]$. Of note, we divided our stalk only invasive cases into focal (80\%) and extensive (20\%) categories similar to base invasive cases, but outcomes were identical and thus separating the stalk only may not be justified. This observation supports our hypothesis of compositional, anatomical, and biological differences of stalk versus base stromal invasion, with the latter representing indolent disease successfully treated by TURBT. The significantly higher risk of progression in base extensive tumors accords with their more aggressive phenotype, potentially deeper bladder wall involvement, and understaging on the initial TURBT. Thus, recognizing and differentiating stalk invasive from base focal and base extensive invasion PUC may convey an important message to clinicians for optimal further disease management. 
The strength of our study design is in very stringent selection criteria whereby we focused on primary PUC, eliminating pT1 cases of non-papillary solid architecture, cases with concurrent CIS, lymphovascular invasion, absent muscularis propria, or clinically advanced disease. With few exceptions, the majority of other pT1 substaging studies have lumped together papillary and non-papillary pT1 tumors, as well as pT1 tumors with and without concurrent CIS as a "total inclusion" routine to maximize numbers of cases $[1,2,7,9,10$, 12, 19, 20, 25, 26]. Evidently, pT1 cancers of solid architecture arising from CIS are biologically different from papillary pT1, more frequently exhibit infiltrative versus nodular growth pattern, and portend significantly higher risk of progression [19, 26]. Presence of associated CIS further contributes to tumor aggressiveness [2, 15, 19]. Secondly, our substaging approach is more easily applied to daily practice with group separation based on morphologic appearance of stroma and 20x objective for size and multifocality assessment rather than laborious morphometric measurements $[6,10,12]$ or ancillary immunohistochemical staining $[27,28]$. However, as a result of strict selection criteria and low incidence of stalk only PUC, the numbers of patients in our study are relatively low. Excluding urothelial carcinomas with non-papillary architecture also reduces case numbers and represents a limitation of our study. With combined efforts from multiple institutions, it will be important to further validate the reproducibility of our proposed scheme in a larger prospective cohort.

In summary, we proposed an innovative, practical pT1 substaging system for PUC and showed that site and extent of lamina propria invasion are significantly associated with risks of progression and prognosis. Tumor invasion limited to the papillary stalk clearly represents morphologically and prognostically indolent group and is worth reporting separately from the base invasive tumors due to their minimal risk of progression. In contrast, reporting of extensive base invasion as a significant adverse prognostic factor may be important in identifying patients most likely to benefit from more aggressive treatment.

\section{Supplementary Material}

Refer to Web version on PubMed Central for supplementary material.

\section{Acknowledgments}

This work was partially supported by the National Cancer Institute at the National Institutes of Health [P50 CA097186 (RG)].

\section{References}

1. Patriarca C, Hurle R, Moschini M, et al. Usefulness of pT1 substaging in papillary urothelial bladder carcinoma. Diagn Pathol. 2016; 11:6,016-0466-6. [PubMed: 26791567]

2. van Rhijn BW, Burger M, Lotan Y, et al. Recurrence and progression of disease in non-muscleinvasive bladder cancer: from epidemiology to treatment strategy. Eur Urol. 2009; 56:430-42. [PubMed: 19576682]

3. Babjuk M, Bohle A, Burger M, et al. EAU Guidelines on Non-Muscle-invasive Urothelial Carcinoma of the Bladder: Update 2016. Eur Urol. 2016

4. Edge, SB., Byrd, DB., Compton, CC., Fritz, AG., Greene, FL., Trotti, A. AJCC cancer staging handbook. 7. 2010. 
5. Moch, H., Humphrey, PA., Ulbright, TM., Reuter, VE. WHO Classification of Tumours of the Urinary System and Male Genital Organs. 4. 2016.

6. Cheng L, Weaver AL, Neumann RM, Scherer BG, Bostwick DG. Substaging of T1 bladder carcinoma based on the depth of invasion as measured by micrometer: A new proposal. Cancer. 1999; 86:1035-43. [PubMed: 10491531]

7. Hermann GG, Horn T, Steven K. The influence of the level of lamina propria invasion and the prevalence of p53 nuclear accumulation on survival in stage T1 transitional cell bladder cancer. J Urol. 1998; 159:91-4. [PubMed: 9400444]

8. van der Aa MN, van Leenders GJ, Steyerberg EW, et al. A new system for substaging pT1 papillary bladder cancer: a prognostic evaluation. Hum Pathol. 2005; 36:981-6. [PubMed: 16153461]

9. Orsola A, Trias I, Raventos CX, et al. Initial high-grade T1 urothelial cell carcinoma: feasibility and prognostic significance of lamina propria invasion microstaging $(\mathrm{T} 1 \mathrm{a} / \mathrm{b} / \mathrm{c})$ in BCG-treated and BCGnon-treated patients. Eur Urol. 2005; 48:231, 8. discussion 238. [PubMed: 15963635]

10. Brimo F, Wu C, Zeizafoun N, et al. Prognostic factors in T1 bladder urothelial carcinoma: the value of recording millimetric depth of invasion, diameter of invasive carcinoma, and muscularis mucosa invasion. Hum Pathol. 2013; 44:95-102. [PubMed: 22939956]

11. Pan CC. Does muscularis mucosae invasion in extensively lamina propria-invasive high-grade papillary urothelial carcinoma provide additional prognostic information? Am J Surg Pathol. 2013; 37:459-60. [PubMed: 23388127]

12. Hu Z, Mudaliar K, Quek ML, Paner GP, Barkan GA. Measuring the dimension of invasive component in pT1 urothelial carcinoma in transurethral resection specimens can predict time to recurrence. Ann Diagn Pathol. 2014; 18:49-52. [PubMed: 24370460]

13. Esrig D, Freeman JA, Stein JP, Skinner DG. Early cystectomy for clinical stage T1 transitional cell carcinoma of the bladder. Semin Urol Oncol. 1997; 15:154-60. [PubMed: 9394910]

14. Kulkarni GS, Hakenberg OW, Gschwend JE, et al. An updated critical analysis of the treatment strategy for newly diagnosed high-grade T1 (previously T1G3) bladder cancer. Eur Urol. 2010; 57:60-70. [PubMed: 19740595]

15. Martin-Doyle W, Leow JJ, Orsola A, Chang SL, Bellmunt J. Improving selection criteria for early cystectomy in high-grade t1 bladder cancer: a meta-analysis of 15,215 patients. J Clin Oncol. 2015; 33:643-50. [PubMed: 25559810]

16. Emiliozzi P, Pansadoro A, Pansadoro V. The optimal management of T1G3 bladder cancer. BJU Int. 2008; 102:1265-73. [PubMed: 19035891]

17. Saito W, Amanuma M, Tanaka J, Heshiki A. Histopathological analysis of a bladder cancer stalk observed on MRI. Magn Reson Imaging. 2000; 18:411-5. [PubMed: 10788718]

18. Lopez-Beltran A, Cheng L. Stage pT1 bladder carcinoma: diagnostic criteria, pitfalls and prognostic significance. Pathology. 2003; 35:484-91. [PubMed: 14660098]

19. Orsola A, Werner L, de Torres I, et al. Reexamining treatment of high-grade T1 bladder cancer according to depth of lamina propria invasion: a prospective trial of 200 patients. Br J Cancer. 2015; 112:468-74. [PubMed: 25535728]

20. Soukup V, Duskova J, Pesl M, et al. The prognostic value of T1 bladder cancer substaging: a single institution retrospective study. Urol Int. 2014; 92:150-6. [PubMed: 24481124]

21. Paner GP, Ro JY, Wojcik EM, Venkataraman G, Datta MW, Amin MB. Further characterization of the muscle layers and lamina propria of the urinary bladder by systematic histologic mapping: implications for pathologic staging of invasive urothelial carcinoma. Am J Surg Pathol. 2007; 31:1420-9. [PubMed: 17721199]

22. Chang WC, Chang YH, Pan CC. Prognostic significance in substaging ofT1 urinary bladder urothelial carcinoma on transurethral resection. Am J Surg Pathol. 2012; 36:454-61. [PubMed: 22261706]

23. Tommasini-Degna A, Negri R. Neoplastic invasion of the connective stalks in transitional cell papillary tumors (TCPT) of the bladder. Urol Res. 1987; 15:31-4. [PubMed: 3824712]

24. Jimenez RE, Keane TE, Hardy HT, Amin MB. pT1 urothelial carcinoma of the bladder: criteria for diagnosis, pitfalls, and clinical implications. Adv Anat Pathol. 2000; 7:13-25. [PubMed: 10640198] 
25. Bertz S, Denzinger S, Otto W, et al. Substaging by estimating the size of invasive tumour can improve risk stratification in pT1 urothelial bladder cancer-evaluation of a large hospital-based single-centre series. Histopathology. 2011; 59:722-32. [PubMed: 22014053]

26. Denzinger S, Burger M, Fritsche HM, et al. Prognostic value of histopathological tumour growth patterns at the invasion front of T1G3 urothelial carcinoma of the bladder. Scand J Urol Nephrol. 2009; 43:282-7. [PubMed: 19382004]

27. Mhawech P, Iselin C, Pelte MF. Value of immunohistochemistry in staging T1 urothelial bladder carcinoma. Eur Urol. 2002; 42:459-63. [PubMed: 12429154]

28. Paner GP, Brown JG, Lapetino S, et al. Diagnostic use of antibody to smoothelin in the recognition of muscularis propria in transurethral resection of urinary bladder tumor (TURBT) specimens. Am J Surg Pathol. 2010; 34:792-9. [PubMed: 20421781] 

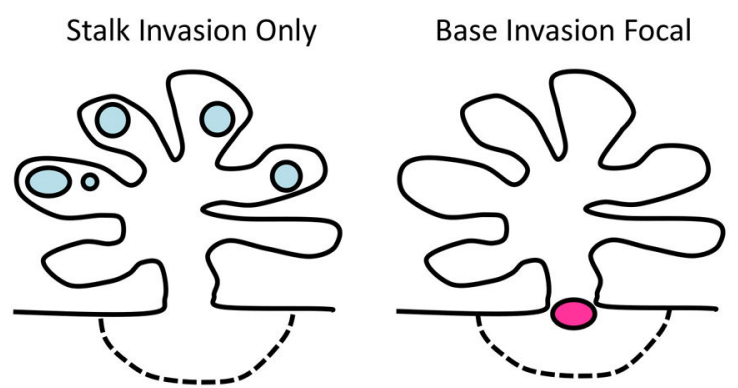

Base Invasion Extensive
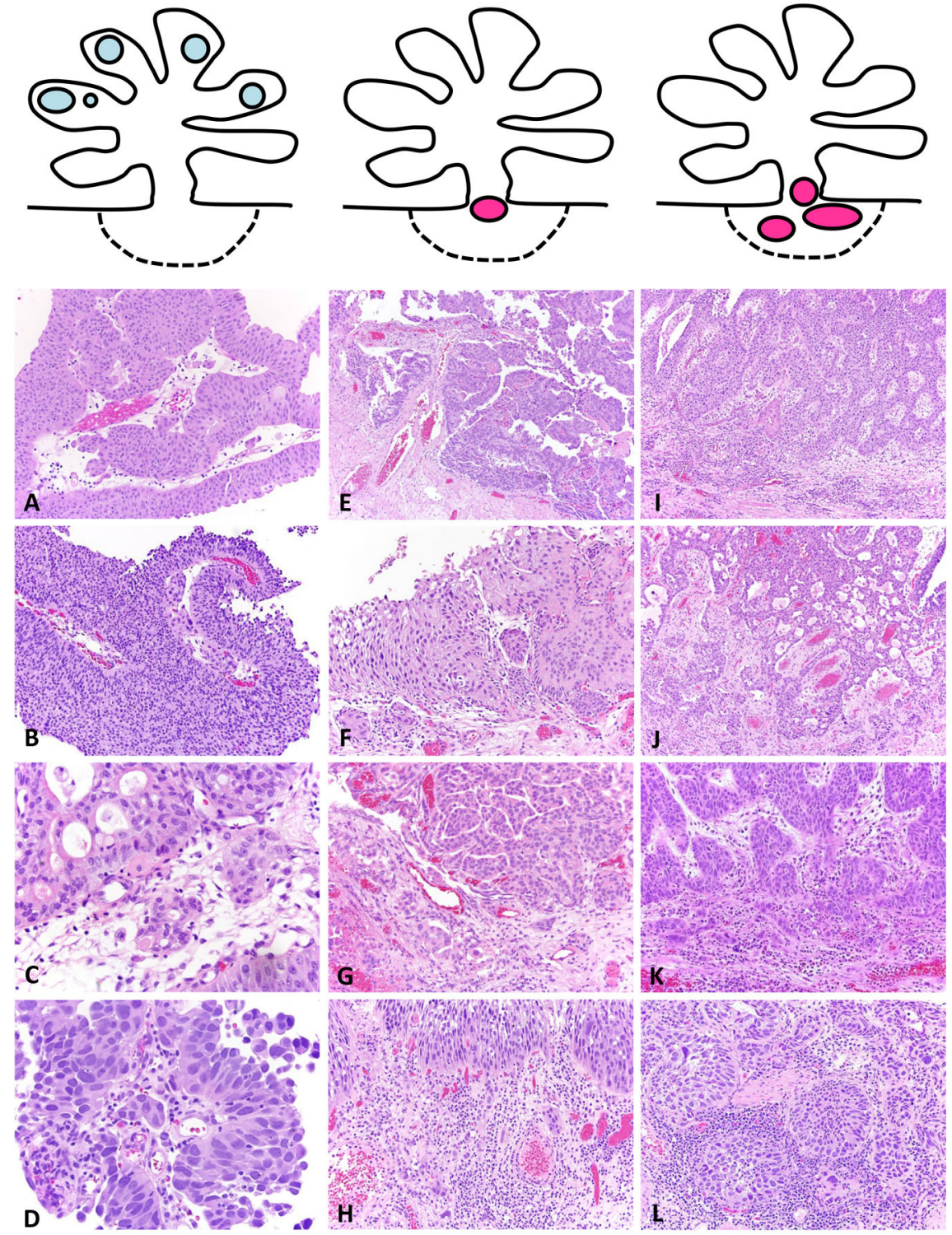

Figure 1.

Different levels of lamina propria invasion in papillary urothelial carcinomas (pT1) demonstrated on eighteen representative study cases. Stalk invasion (A-D) is characterized by presence of single cells, variably sized rounded outpouchings, or irregular tumor nests confined to the papillary stalk of loose edematous connective stroma, often with paradoxical differentiation and retraction artefact. In contrast to stalk, base connective stroma (E-L) was more condensed, eosinophilic, with obvious muscularis mucosa fascicles, prominent vasculature, increased inflammatory infiltrate, and sometimes marked desmoplastic response. The base focal category was defined as a single focus of microinvasion within 1 high power field of view (HPF) at $20 \times$ corresponding to $1 \mathrm{~mm}$ area $(\mathrm{E}-\mathrm{H})$. The base extensive was defined as either few microinvasive foci more than $1 \mathrm{~mm}$ apart, present in 
multiple tissue fragments, or invasion of area that would not fit within one $20 \times \mathrm{HPF}$ (I-L). Original magnification is $100 \times(\mathrm{E}, \mathrm{I}, \mathrm{J}), 200 \times(\mathrm{A}, \mathrm{B}, \mathrm{F}, \mathrm{G}, \mathrm{H}, \mathrm{K}, \mathrm{L}), 400 \times(\mathrm{C}, \mathrm{D})$. 

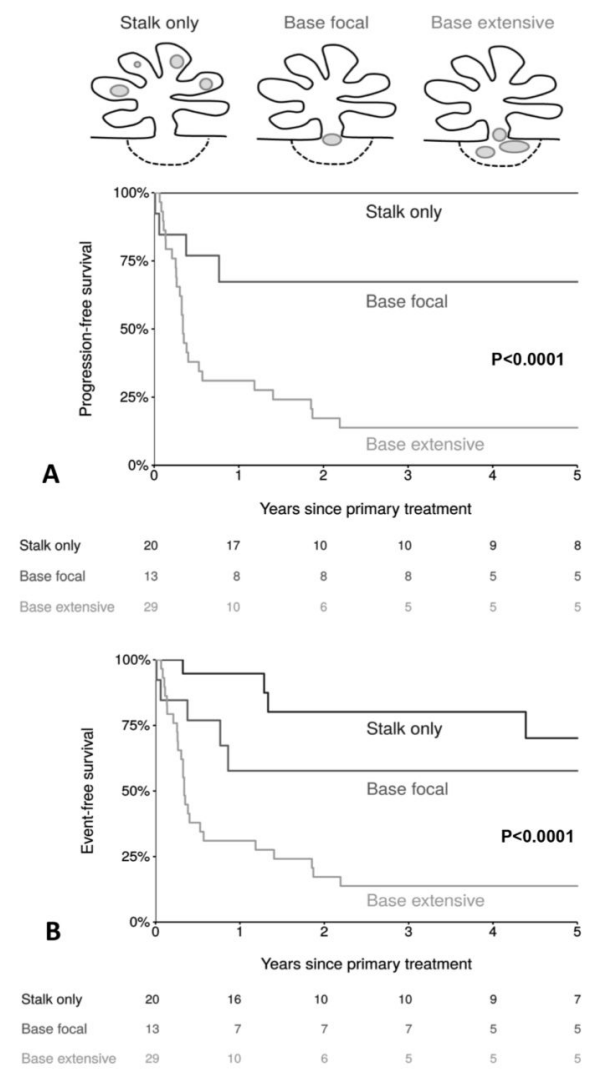

Figure 2.

Kaplan-Meier estimates of progression-free (A) and event-free (B) survival curves by tumor subtype. Progression-free survival was defined as time from primary treatment to first documented progression event (stage progression or multifocality with or without concurrent CIS) with at other-cause death or last follow-up. Event-free survival was defined as time from primary treatment to the earlier of first documented progression event and first documented recurrence event (any non-muscle invasive papillary cancer including pTa or pT1) with censoring at other-cause death or last follow-up. 


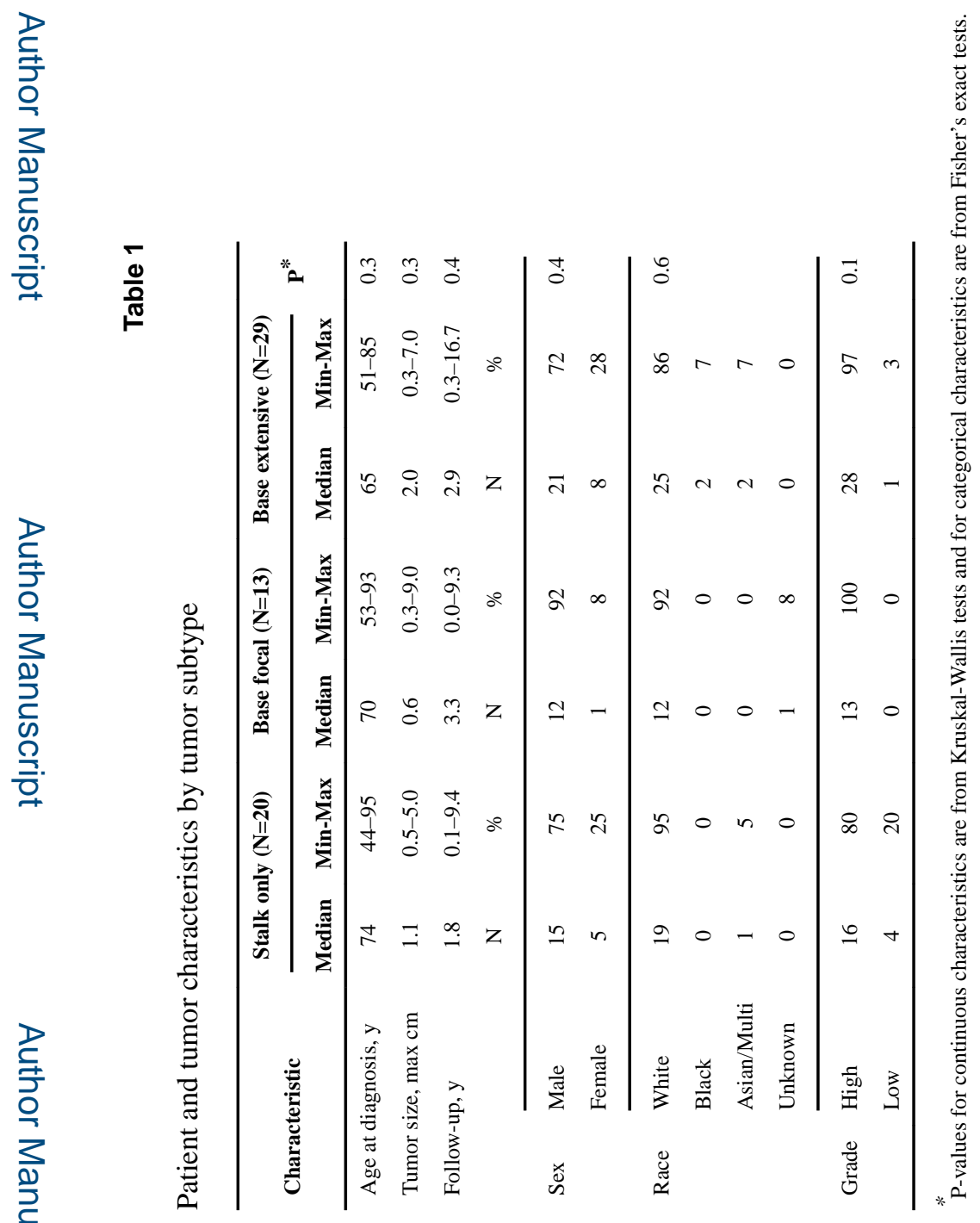




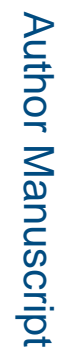

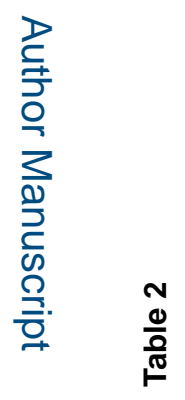

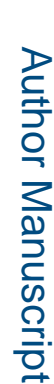

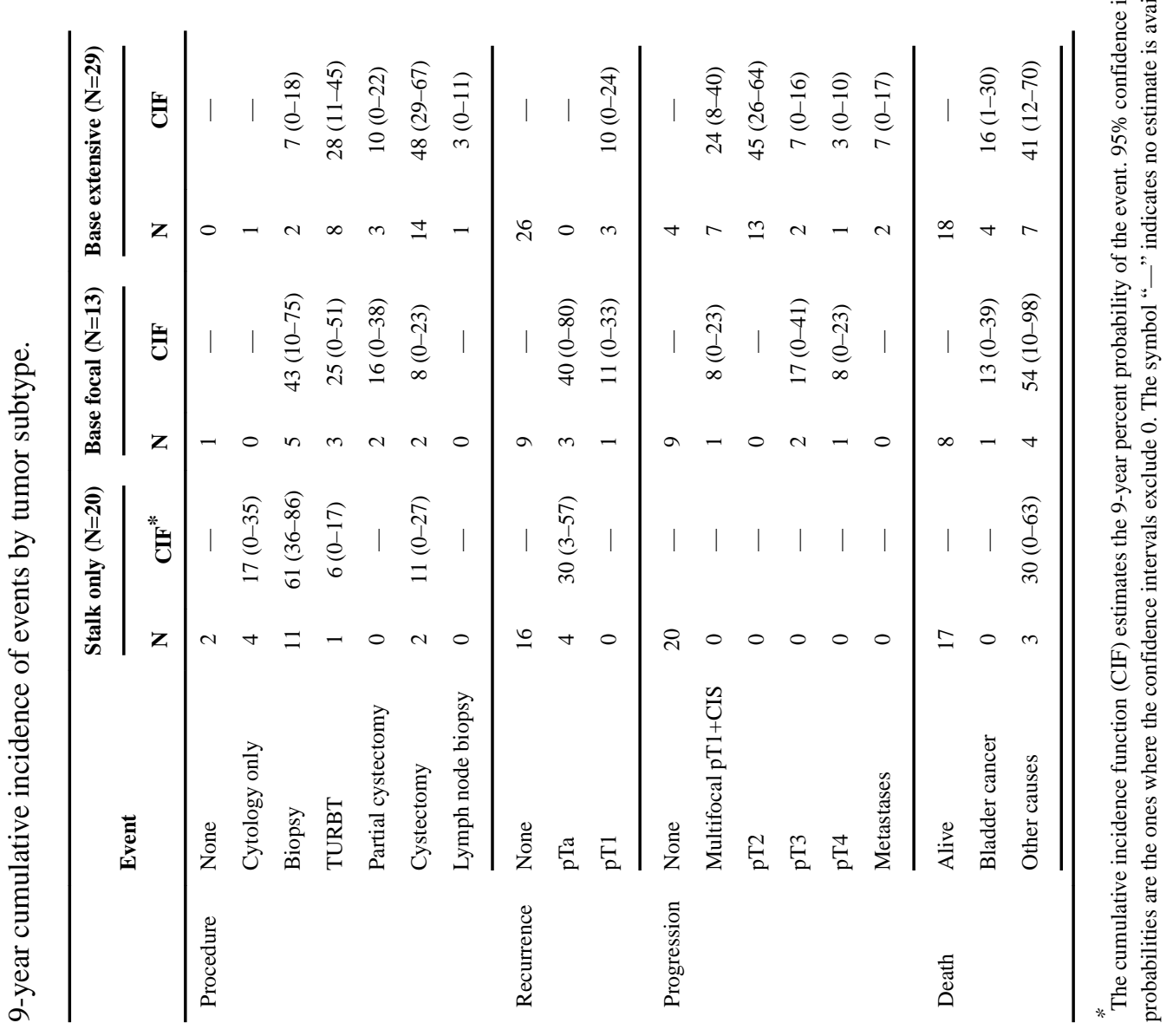

Histopathology. Author manuscript; available in PMC 2018 September 01. 


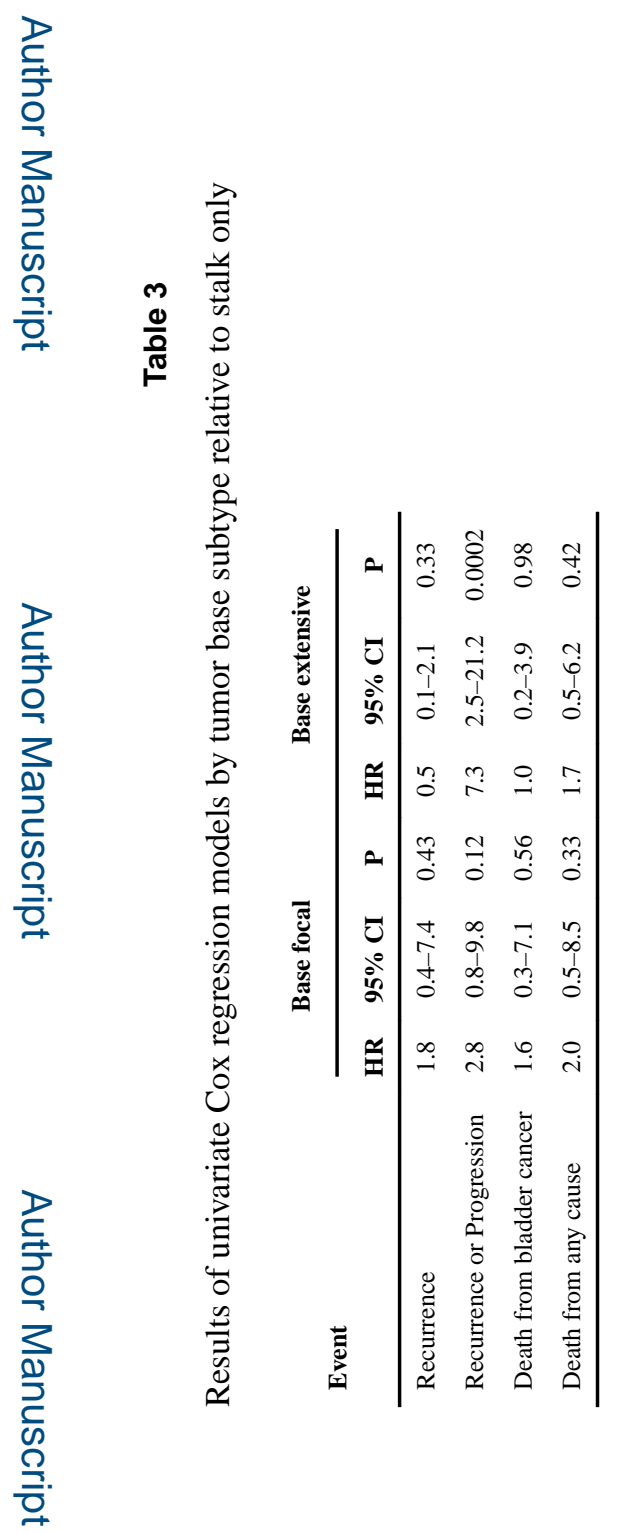

Histopathology. Author manuscript; available in PMC 2018 September 01. 